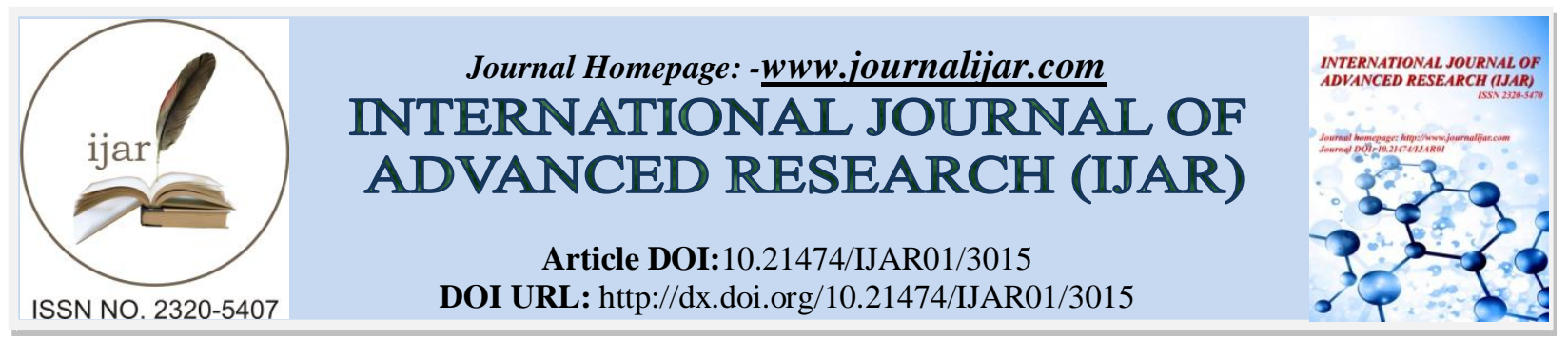

RESEARCH ARTICLE

\title{
Awareness of Colorectal Cancer among Inflammatory Bowel Disease Patients in a Tertiary Care Hospital: An Epidemiological STUdy.
} Leenah Saad Al Ahmari, Hadel Sultan Al Subaie, Jumanah Yousef Al Shenaifi, Reema Ayed Al Anazi,
Othman Rabeh Al Harbi, Firas Ghomraoui.

\section{Manuscript Info}

Manuscript History

Received: 29 November 2016

Final Accepted: 27 December 2016

Published: January 2017

\section{Abstract}

Background:Colorectal cancer (CRC) is documented to be the third most common cancer worldwide in terms of incidence and mortality.Inflammatory bowel disease (IBD) patients are at higher risk for developing CRC as compared to the general population, as CRC is the most common cancer in the IBD population. This study aims to assessthe awareness of CRC amongIBD patients in Saudi Arabia.

Methods:A quantitative, observational, retrospective cohortstudy was conducted at King Khalid University Hospital(KKUH), in Riyadh, Saudi Arabia betweenNovember 2013 and February 2014.Directedinterview questionnaires were administered over the telephone to the 384 purposively-selected IBD-established patients.

Results:Prompted awareness of CRC symptoms and risk factors was much higher than unprompted awareness. When asked about risk factors, nearly $48 \%$ of patients could not recall any risk factorsunaided. Overall, the most commonly identified risk factor for CRC unaided was IBD $(27.1 \%)$, followed by diet (20.5\%).However,14.5\% couldnot identify or didnot know whether their own disease (IBD) is a risk factor for CRC.

Conclusion:Results from thisstudy showed that the overall awareness of CRC symptoms and risk factors among Saudi IBD patients was poor. However, the knowledge towards CRC symptoms was observed higher than knowledge of risk factors, insinuating a possible gap in patient education. Customization of health-promotion programs for IBD patients is urgently needed to combat the rising incidence of CRC amongst them.

Copy Right, IJAR, 2016,. All rights reserved.

\section{Introduction:-}

Colorectal cancer is a common cancer and is ranked as the third in males and the second in females worldwide. ${ }^{1}$ In Saudi Arabia, CRC frequency is documented as second to breast cancer. This rate is alarmingly increasing and is estimated to leap up to a fourfold increase by the year $2030^{2}$. Furthermore, it is also reported to be the third leadingcause of cancer death in the United States. ${ }^{3}$

Several risk factors were proven to be associated with the development of CRC. Modifiable factors include obesity, sedentary lifestyle, cigarette smoking, alcohol intake, red meat ingestion, and decreased vegetable and fruit 
consumption. On the other hand, non-modifiable factors constitute the following: Family history of CRC, older age and inflammatory bowel disease (IBD), which falls under the scope of this study. ${ }^{4}$

Previous studies assessing the awareness of CRC in several aspects, including epidemiology, symptoms/signs, and risk factors have revealed a poverty in the knowledge of the public, which is disconcerting given the fact that CRC still remains largely a topic of confusion in the general population.This has contributed significantly to the deleterious consequences on time of presentation and, therefore, on overall survival. ${ }^{5-11}$ These findings were also consistent with the data of another study, which showed that the level of CRC knowledge among the Saudi general public was low, thus signifying the necessity of continuing public education and screening programs. ${ }^{12}$ Furthermore, the lack of awareness was not only reported among the general public, but even more among patients following up in thegastroenterology clinics and the peripheral colorectal surgical unit. ${ }^{13,14}$

This issue needs to be taken seriously since it has been demonstrated that blurred recognition of CRC susceptibly among high-risk groups does exist. One study investigated the awareness of the potential risk for developing CRC in 195 overweight patients where only a third out of them could identify their weight as a risk factor. ${ }^{15}$

This, subsequently, provoked the interest to assess the awareness of CRC in a subset of the general population, IBD patients. This is due to the fact that these patients are at higher risk for developing CRC as compared to the general population and that CRC is found to be the most common cancer among them. ${ }^{6}$

Despite this, we are not aware of any studies addressing this issue locally or internationally. This adds to the uniqueness and indispensability of our study. Since we believe that assessing CRC awareness among IBD patients will help estimating the real magnitude of this issue which will draw the attention of decision-makers and healthcare professionals to increase the efforts made for educating the patients and subsequently, lead to earlier presentation and improved survival rates.

\section{Methods:-}

A quantitative, observational, retrospective cohort study was carried out to assess the awareness of CRC among IBD patients in King Khalid University Hospital (KKUH) in the periods between November 2013 and February 2014. Patients who were below the age of 12 or have had a history of CRC and/or colonic polyps were excluded from the study. 800 patients were present in the local IBD database compiled between the periods of 2009 and 2013, of which $580(72.5 \%)$ were eligible to be enrolled into the study according to the exclusion criteria.Directed-interview questionnaires were administered to those eligible over the telephone to gather current data pertaining to their awareness. Of those, 317 (54.7\%)responded to this study. The questionnaires were compiled and modified based on a pilot study conducted on 12 patients, and consisted in its final form of 16 questions that covered several variables, includingdemographics, family history of CRC, and overall knowledge of the disease (risk factors, signs/symptoms).

\section{Data analysis:-}

Collected data was entered to Excel version 14.00 and categorical variables were expressed as frequencies.Data was analyzed using SPSS version 21. (SPSS Inc., Chicago, IL, USA) and all statistical tests were declared significant at level of 0.05 or less. Chi-square tests were used to examine differences in CRC awareness in relation to sociodemographic factors including gender, age, diagnosis, marital status, occupation, education level, income and having a diagnosed relative or friend of CRC.

\section{Ethical considerations:}

Confidentiality with regards to patients' information was maintained and participants' consent was obtained verbally over the telephone. All patients were informed about the purpose of the research, the reason why they were chosen and their right to discontinue from participation at any time without being obligated by the investigators. Neither rewards nor incentives were given to the patients. No conflict of interest was present.

\section{Results:-}

\section{Socio-Demographic Characteristics:-}

Of the 580 IBD patients who were eligible to enroll, 317 (54.7\%) responded to the study. A total of 317 IBD patients were interviewed over the telephone (Table 1). 55.2\%had Crohn's disease (CD) while 44.6\% were diagnosed with ulcerative colitis (UC) based on endoscopic criteria. $55.5 \%$ were male while $44.5 \%$ were female, 
with a mean age of(Add mean/SD of age here) Patients less than thirty years of age formed a proportion of 55.3\% while older patients ( $\geq 30$ years) formed the remaining group. $96.5 \%$ were Saudis, where those who reside in Riyadh formed the largest proportion of the respondents. $79.5 \%$ of the IBD patients have attained either a high school or university degree while $20.5 \%$ were fromother educational levels (illiterate, reads \& write, primary school, middle school and higher studies).60.6\% were either governmental employees or students. $52.4 \%$ of the patients were married. The monthly income of $80 \%$ of the patients was ranging from 1,333 or less to 5,333USD.The vast majority had no first or second-degree relatives nor did they have any friends diagnosed with CRC $(90.5 \%$ and $80.1 \%$, respectively).

\section{Knowledge of CRC Risk Factors, Signs andSymptoms:-}

Responses to knowledge assessments including recognition of risk factors and signs and symptoms were variable (See tables $2 \& 3$ ). Out of the 317 patients, 35.3\% have never heard of CRC and $62.1 \%$ of the patients either don't think or don't know whether CRC is common or not. When asked about sources of information, the internet was the most popular method used by IBD patients to learn more about CRC while doctors' education was the second most commonly chosen source of information (Chart 1).

\section{Knowledge of Risk Factors:-}

In questions relating to patients' knowledge of CRC risk factors, 59\% of IBD patients answered "no" to the question "do you know any risk factors of colorectal cancer?"

Nearly $48 \%$ of patients could not recall any risk factors without aided while $45.5 \%$ of them still didn't manage to recall and answered "no" or "I don't know" after being prompted.

Overall, the most commonly identified risk factor for CRC unaided was IBD (27.1\%) and it was followed by diet $(20.5 \%)$. Out of all patients, $14.5 \%$ couldn't recognize or didn't know whether their own disease (IBD) is a risk factor for CRC. On the other hand, aid was needed by more than half of the patients (58.4\%) to identify IBD as a risk factor. The least well-recognized risk factors for CRC, however, were by far diabetes mellitus $(57.1 \%)$ and hypertension (53.9\%). Similarly, Age and gender were not recognized by around half of the patients $(50.8,54.3 \%$, respectively).

Most risk factors were correctly chosen by patients after aided, these include alcohol (66.6\%), smoking (63.7\%), family history $(57.7 \%)$ and obesity $(47.3 \%)$.

However, a proportion of $54.6 \%$ of the IBD patients incorrectly chosehemorrhoids as a risk factor for CRC after aided.

\section{Knowledge of Symptoms:-}

Around $50 \%$ of IBD patients believed they don't know any symptoms while approximately a similar proportion of them answered "yes" to the question "do you know any symptoms of colorectal cancer?". Abdominal pain was the most frequent symptom of CRC that was correctly believed by $28.7 \%$ of the patients without being prompted while $7.6 \%$ managed to name "weight loss" as a symptom of CRC. Other symptoms of CRC were answered correctly by the patients only after being aided, these include bleeding perrectum (53.9\%) and change in bowel habits (59\%). Difficulty of swallowing was correctly judged by $54.9 \%$ of IBD patients not to be a symptom of CRC. However, black stools (17\%) and fatigue (14.5\%) were the most frequent wrongly identified as not being symptoms of CRC after being prompted.

Around $50 \%$ of IBD patients correctly answered "yes" to the question "do you think that colorectal cancer can present without symptoms?" while about $39 \%$ of patients believed the opposite.

\section{Factors Associated With Knowledge of CRC Signs, Symptoms and Risk Factors:-}

A confidence interval of $95 \%$ was used and results showed that men demonstrated higher unprompted knowledge of risk factors than women, they were more likely to recognize IBD $(\mathrm{p}=0.056)$ and smoking $(\mathrm{p}=0.015)$ as risk factors for CRC. Older patients were also better at identifying symptoms of CRC compared to the younger age group. For example, those aged 30 or older correctly named that bleeding per rectum and fatigue are symptoms of CRC ( $\mathrm{p}=0.038, \mathrm{p}=0.026$ respectively). Moreover, they could also identify obesity as a possible risk factor for CRC without being aided $(\mathrm{p}=0.016)$. On the other hand, most patients who are less than 30 years of age wrongly believed 
that CRC is not common compared to older patients $(\mathrm{p}=0.001)$. Patients who had university or higher study education showed higher recognition of symptoms and risk factors without being aided, they correctly identified bleeding per rectum as a symptom $(p=0.008)$ and IBD $(p=0.006)$, family history $(p=0.017)$ as risk factors for CRC.The different knowledge levels betweenCD and UC patients werestatisticallyinsignificant.

\section{Discussion:-}

Determining the IBD patients' awareness regarding CRC may offer significant information to incorporate the policy decision for screening promotion, early diagnosis and reduction of morbidity and mortality of CRC.Until now, and according to the available literature, this is the first study to assess the awareness of CRC among a cohort of IBD patients. This study showed that a large proportion of patients had poor awareness regarding symptoms and risk factors of CRC. Prompted awareness of CRC symptoms and risk factors was much higher than unprompted awareness which was also reported in an earlier study that was conducted among the general public of the United Kingdom. ${ }^{5}$ Our study also shows that Saudi IBD patients were more aware of CRC symptoms than risk factors, this could be justified by the resemblance of presenting symptoms between CRC and IBD which might have confused and aided them in reaching a more rightful prediction.

When comparing the proportion of Saudi IBD patients who could recognize their own disease (IBD) as a risk factor for CRC after being aided to the general British population ${ }^{5}$, it is striking to say that the Saudi IBD patients' awareness to IBD as a risk factor is not that different from the general public but in fact less $(58.4 \%$ and $61.9 \%$, respectively) despite that they were expected to be better informed than average about CRC. This is worrisome since it implies that Saudi IBD patients are either experiencing poor public health educational programs or in denial to the fact that their risk is higher compared to the general population.

It is worth mentioning that IBD was the only common recognized risk factor among both the Saudi general public and within the Saudi IBD population possessing higher levels of education, which mayguide the focus of the educational efforts to the other less identified risk factors. ${ }^{12}$

The most recalled symptom of CRC without prompt by Saudi patients was abdominal pain. Likewise, a Malaysian study among rural population reported that abdominal pain was the most frequent symptom identified unaided and the second most agreed symptom of CRC from prompted question. ${ }^{10}$ Weight loss, however, was the least recognized symptom of CRC unaided among the Saudi IBD patients in this study, perhaps due to the fact that this symptom is non-specific to CRC and is not anatomically confined to the abdomen (as in the case of abdominal pain). This might compromise the professional help-seeking behaviors. Similarly, the same Malaysian study demonstrated that only less than $5 \%$ could relate weight loss to CRC without aided. ${ }^{10}$

Men knew more CRC risk factors than women, particularly smoking and IBD. This could be because men are aware that $\mathrm{CRC}$ is more pronounced among them or perhaps implies men's preference to think that cancer risk is modifiable in regards to smoking. Our results were in disagreement with a couple of studies including alocal study among the general public that revealed a better awareness of CRC symptoms among women. ${ }^{5,12}$ The fact that Saudi men in our study showed higher awareness is encouraging especially that previous studies have also shown that CRC is more common among Saudi males. ${ }^{17,18}$

Older patients showed higher knowledge of symptoms than younger patients especially in bleeding per rectum and fatigue, they have also shown better knowledge in some risk factors like obesity which is reassuring because they are at higher risk and in need to correctly recognize these symptoms and risk factors. Moreover, local studies have reported increased prevalence of CRC among older age groups. ${ }^{18}$ However, interestingly, younger age group $(<30$ years) were more aware of the link between CRC and alcohol than older patients. This is consistent with findings from the same British study which reported that younger age groups had a higher awareness of alcohol as a risk factor for CRC. ${ }^{5}$

Diet was a well-recognized risk factor by the patients after aid and this provides a positive implication due to the predominance of unhealthy lifestyle habits among the Saudi population, for example being overweight ${ }^{19}$, poor fruit and vegetable, high meat and fat consumption.

Knowing someone with CRC or having a family history of CRC was present in very few patients in this study which hindered the process of finding any statistical significance. 
Certain limitations of this study should be noted.The method of data collection used in this study was designed to offer a straightforward evaluation of knowledge levels, it hadn't been previously validated and therefore cannot be used for international comparison of CRC awareness. Moreover, due to the fact that our study is a single-center study and that most of our patients were Riyadh citizens receiving good level of education, findings may not necessarily be applicable to rural hospitals in Saudi Arabia.

\section{Conclusion:-}

Saudi IBD patients' awareness of CRC symptoms and risk factors is alarmingly low, emphasizing the significance of the health-care professionals to continue educating them about their own risk of CRC and the need of more solid screening programs which will lead to the reduction of CRC incidence and mortality. Moreover, customization of health-promotion programs for IBD patients is noteworthy in order to increase their CRC awareness. Furthermore, since internet was the most frequented source of information, the need of establishing a well-structured website is indispensable. Lastly, further research should be conducted with validated questionnaires to permit comparison of awareness level of CRC among IBD patients worldwide.

\section{Acknowledgments:-}

We would like to express our deep gratitude to Dr. ShaffiAhamed and Mr. Philip Feeleywho were of most assistance to us in regards to statistical analysis. We would also like to convey our gratitude to the medical students who participated in collecting the data for our study.

\section{References:-}

1. Jemal A, Bray F, Center M, Ferlay J, Ward E, Forman D. Global cancer statistics. CA Cancer J Clin. 2011;61(2):69-90.

2. Ibrahim EM, Zeeneldin AA, El-Khodary TR, Al-Gahmi AM, Bin Sadiq BM. Past, present and future of colorectal cancer in the Kingdom of Saudi Arabia. Saudi J Gastroenterol. 2008 Oct;14(4):178-82.

3. Siegel R, Naishadham D, Jemal A. Cancer statistics, 2013. CA Cancer J Clin. 2013 Jan;63(1):11-30.

4. Johnson CM, Wei C, Ensor JE, Smolenski DJ, Amos CI, Levin B, Berry DA. Meta-analyses of colorectal cancer risk factors. Cancer Causes Control. 2013 Jun;24(6):1207-22.

5. Power E, Simon A, Juszczyk D, Hiom S, Wardle J. Assessing awareness of colorectal cancer symptoms: measure development and results from a population survey in the UK. BMC Cancer. 2011 Aug;23(11):366.

6. Manning AT, Waldron R, Barry K. Poor awareness of colorectal cancer symptoms; a preventable cause of emergency and late stage presentation. Ir J Med Sci. 2006 Oct-Dec;175(4):55-7.

7. Breslow R, Sorkin J, Frey C, Kessler L. Americans' knowledge of cancer risk and survival. Prev Med. 1997;26(2):170-177.

8. Gimeno-García AZ, Quintero E, Nicolás-Pérez D, Jiménez-Sosa A. Public awareness of colorectal cancer and screening in a Spanish population. Public Health. 2011 Sep;125(9):609-15.

9. Keighley MR, O'Morain C, Giacosa A, Ashorn M, Burroughs A, Crespi M, et al; United European Gastroenterology Pederation Public Affairs Public awareness of risk factors and screening for colorectal cancer in Europe. Eur J Cancer Prev. 2004 Aug;13(4):257-62

10. Su T, Goh J, Tan J, Muhaimah A, Pigeneswaren Y, Khairun N, et al. Level of colorectal cancer awareness: a cross sectional exploratory study among multi-ethnic rural population in Malaysia. BMC Cancer. 2013 Aug $7 ;(13): 376$.

11. Christou A, Thompson SC. Colorectal cancer screening knowledge, attitudes and behavioural intention among Indigenous Western Australians. BMC Public Health. 2012 Jul 18;(12):528.

12. Zubaidi AM, AlSubaie NM, AlHumaid AA, Shaik SA, AlKhayal KA, AlObeed OA. Public awareness of colorectal cancer in Saudi Arabia: A survey of 1070 participants in Riyadh. Saudi J Gastroenterol. 2015 MarApr;21(2):78-83.

13. Harewood GC, Murray F, Patchett S, Garcia L, Leong WL, Lim YT, et al. Assessment of colorectal cancer knowledge and patient attitudes towards screening: is Ireland ready to embrace colon cancer screening? Ir J Med Sci. 2009 Mar;178(1):7-12.

14. McVeigh TP, Lowery AJ, Waldron RM, Mahmood A, Barry K. Assessing awareness of colorectal cancer symptoms and screening in a peripheral colorectal surgical unit: a survey based study. BMC Surg. 2013 Jun 22;13(1):20. 
15. Leite-Pereira F, Medeiros R, Dinis-Ribeiro M. Overweight and obese patients do not seem to adequately recognize their own risk for colorectal cancer. J Cancer Educ. 2011 Dec;26(4):767-73.

16. Forbes GM. Colorectal cancer screening tests: pros and cons, and for whom? Expert Rev GastroenterolHepatol. 2008 Apr;2(2):197-205.

17. Al-Ahwal MS, Shafik YH, Al-Ahwal HM. First national survival data for colorectal cancer among Saudis between 1994 and 2004: what's next? BMC Public Health. 2013 Jan 25;13:73.

18. Mosli MH, Al-Ahwal MS. Does the increasing trend of colorectal cancer incidence in jeddah reflect a rise in the Kingdom of Saudi Arabia? Asian Pac J Cancer Prev. 2012;13(12):6285-8.

19. Ng SW, Zaghloul S, Ali HI, Harrison G, Popkin BM. The prevalence and trends of overweight, obesity and nutrition-related non-communicable diseases in the Arabian Gulf States. Obes Rev. 2011 Jan;12(1):1-13. 